\title{
On the analysis of stability, bifurcation, and chaos control of discrete-time predator-prey model with Allee effect on predator
}

\author{
Seval Işık ${ }^{* 1}$ (D), Figen Kangalgil ${ }^{2}$ (D) \\ ${ }^{1}$ Sivas Cumhuriyet University, Faculty of Education, Department of Mathematics and Science Education, \\ 58140 Sivas, Turkey \\ ${ }^{2}$ Dokuz Eylul University, Bergama Vocational High School, 35700 Izmir, Turkey
}

\begin{abstract}
In this paper, a discrete predator-prey model with Allee effect which is obtained by the forward Euler method has been investigated. The local stability conditions of the model at the fixed point have been discussed. In addition, it is shown that the model undergoes Neimark-Sacker bifurcation by using bifurcation theory. Then, the direction of NeimarkSacker bifurcation has been given. The OGY method is applied in order to control chaos in considered model due to emergence of Neimark-Sacker bifurcation. Some numerical simulations such as phase portraits and bifurcation figures have been presented to support the theoretical results. Also, the chaotic features are justified numerically by computing Lyapunov exponents. Because of consistency with the biological facts, the parameter values have been taken from literature [Controlling chaos and Neimark-Sacker bifurcation discrete-time predator-prey system, Hacet. J. Math. Stat. 49 (5), 1761-1776, 2020].
\end{abstract}

Mathematics Subject Classification (2020). 39A33, 37G35, 39A30

Keywords. predator-prey model, fixed point, stability, Neimark-Sacker bifurcation, Allee effect, OGY control method

\section{Introduction}

The predator-prey systems which show relationships between two predator-prey species have an important role in both ecology and mathematical biology [8, 19,33]. Comprehending the dynamics of predator-prey models will be very useful for analysis of multiple species interactions. Leslie $[20,21]$ introduced the following famous Leslie predator-prey system where the carrying capacity of the predator is proportional to the number of prey :

$$
\begin{aligned}
& \frac{d N(t)}{d t}=N(t)\left(r_{1}-b P(t)\right), \\
& \frac{d P(t)}{d t}=P(t)\left(r_{2}-\theta \frac{P(t)}{N(t)}\right) .
\end{aligned}
$$

\footnotetext{
*Corresponding Author.

Email addresses: skaracan@cumhuriyet.edu.tr (S. Işı), figen.kangalgil@deu.edu.tr (F. Kangalgil)

Received: 29.04.2020; Accepted: 02.11.2021
} 
where $N(t)$ and $P(t)$ represent population densities of prey and predator at time $t$, respectively. The parameters $r_{1}$ and $r_{2}$ are the intrinsic growth rates of prey and predator, respectively. The parameter $b$ measures the strength of competition among individuals of species $N$. The value $\frac{r_{1}}{b}$ is the carrying capacity of the prey in the absence of predation. The parameter $\theta$ is a measure of the food quantity that the prey provides and is converted to predator birth. The term $\frac{P}{N}$ is called the Leslie-Gower term [18].

When the dynamical systems are modelled, these systems usually take one of two forms: i) continuous-time systems described by differential equations, or ii) discrete-time systems governed by difference equations. Although many authors investigate the nonlinear dynamic characteristics of continuous systems for a long time, the researchers' interest has recently increased to discrete- time systems. The reasons are that these systems provide more efficient computing systems for numerical simulations as compared with continuous ones and also in the case of non-overlapping generation, discrete time systems are more realistic than continuous systems. So, discrete time systems are more attractive than continuous time systems. [1,10,11,13-17,24,28].

The Allee effect phenemenon, which was first explained by Warden Clyde Allee [2] in 1931, describes a positive correlation between any measure of species fitness and population numbers. The main causes of the Allee effect are difficulty in finding mates, inbreeding depression social dysfunction at small population sizes, predator avoidance and food exploitation. In general, Allee effect, which plays an important role in predator-prey models, gives these models a rich dynamic. Many researchers have studied the bifurcation and stability analysis of continuous time models with Allee effect in [4,30,34]. In the literature, a discrete-time models with Allee effect on prey population have been studied intensively, however, the research with Allee effect on predator population is relatively rare $[3,26,31,32]$. So, we introduce a discrete-time system with Allee effect on predator population.

As a parameter is changed, stability may change, emerge or disappear of new stable points. Bifurcation theory is the mathematical work of changes in the qualitative or topological structure of a given continuous or discrete system. Several works $[4,10,12$, 29,30] and the references cited therein have been provided bifurcation and found richer dynamics by doing both theoretical and numerical analysis.

Unpredictable time evolution of many nonlinear systems has been called chaos. There are many different methods and techniques in the literature to ensure chaos control such as OGY method [23,27], time-delay feed-back control method [22] and fuzzy control [9], etc. OGY method which has become more and more interesting in many researchers after introducing by Ott et al. [27] is used in this paper. More detailed information on chaos control can be found in the articles [5-7,23, 25,27].

In the present study motivated by the Leslie predator-prey model, a predator-prey model obtained by adding Allee effect on predator population is considered. By implementing the forward Euler scheme to model (1.1), obtained new model can be written as follow:

$$
\begin{aligned}
& N_{t+1}=N_{t}+\delta N_{t}\left(r_{1}-\varepsilon P_{t}\right) \\
& P_{t+1}=P_{t}+\delta P_{t}\left(r_{2}-\theta \frac{P_{t}}{N_{t}} \frac{P_{t}}{\left(\alpha+P_{t}\right)}\right)
\end{aligned}
$$

where $\delta>0$ is the step size and the term $\frac{P_{t}}{\alpha+P_{t}}$ is Allee effect [34]. $\alpha>0$ is described as a Allee constant. The other parameters are defined as the model (1.1).

Motivated by the above mentioned studies, the contribution and novelty of the paper are presented as follows:

i. The dynamic behavior of a discrete-time predator-prey model obtained by the forward Euler scheme with Allee effect on predator population which is studied rarely is 
investigated. The Allee effect is a new idea in the literature, which gives the model more realistic and complicated. Thus, the model (1.2) has been more realistic compared with model (1.1).

ii. The interior fixed point of the proposed model is found and the topological classification of this fixed point is analysed.

iii. It is shown that the proposed model undergoes Neimark-Sacker bifurcation about its interior fixed point whenever the step size is selected as the bifurcation parameter. In addition, the direction of the Neimark-Sacker bifurcation is given.

iv. The OGY method is applied to the model for controlling chaos due to the emergence of the Neimark-Sacker bifurcation.

v. Finally, three numerical examples are presented for our discrete-time predator-prey model with Allee effect in order to support the accuracy of our theoretical findings.

This paper is organized as follows: In section 1, a discrete predator-prey model is formulated by applying the forward Euler method. In section 2, the topological classifications of the fixed point of the model with Allee effect on predator population are investigated. In section 3, it is shown that the model undergoes Neimark-Sacker bifurcation by using bifurcation theory. Then, the direction of Neimark-Sacker bifurcation is given. In section 4, the OGY method is applied to the model for controlling chaos due to the emergence of the Neimark-Sacker bifurcation. In section 5, in order to verify the analytical findings obtained in the previous sections, three examples are given. Moreover, the bifurcation diagrams, phase portraits and maximum Lyapunov exponents for different parameter values are drawn. Finally, the conclusion is given in section 6 .

\section{Topological classifications of the fixed point of the model (2)}

In this section, we will determine the existence of the fixed point of the discrete-time system and study the stability of this fixed point. To determine the positive fixed point $\left(N^{*}, P^{*}\right)$ we have to solve the following nonlinear system:

$$
\begin{aligned}
N^{*} & =N^{*}+\delta N^{*}\left(r_{1}-\varepsilon P^{*}\right) \\
P^{*} & =P^{*}+\delta P^{*}\left(r_{2}-\theta \frac{P^{*}}{N^{*}} \frac{P^{*}}{\left(\alpha+P^{*}\right)}\right),
\end{aligned}
$$

From the definition of the fixed point, the model (1.2) has one nontrivial fixed point $E^{*}=\left(\frac{r_{1}^{2} \theta}{r_{2} \varepsilon\left(\alpha \varepsilon+r_{1}\right)}, \frac{r_{1}}{\varepsilon}\right)$. The Jacobian matrix of the map (1.2) evaluated at any point $(N, P)$ is given by

where

$$
J=\left(\begin{array}{ll}
a_{11} & a_{12} \\
a_{21} & a_{22}
\end{array}\right)
$$

$$
\begin{array}{ll}
a_{11}=1+\delta r_{1}-\delta P \varepsilon, & a_{12}=-\delta N \varepsilon \\
a_{21}=\frac{\delta P^{3} \theta}{N^{2}(\alpha+P)} & a_{22}=1+\delta r_{2}-\frac{3 \delta P^{2} \theta}{N(\alpha+P)}+\frac{\delta P^{3} \theta}{N(\alpha+P)^{2}} .
\end{array}
$$

The characteristic equation of matrix $J$ can be written as

$$
\lambda^{2}-\operatorname{tr} J \lambda+\operatorname{det} J=0
$$

where $\operatorname{tr} J=a_{11}+a_{22}$ and $\operatorname{det} J=a_{11} a_{22}-a_{12} a_{21}$.

Definition 2.1. A fixed point $(\mathrm{N}, \mathrm{P})$ is called

i) sink if $\left|\lambda_{1}\right|<1$ and $\left|\lambda_{2}\right|<1$, and it is locally asymptotically stable,

ii) source if $\left|\lambda_{1}\right|>1$ and $\left|\lambda_{2}\right|>1$ and it is locally unstable, 
iii) saddle if $\left|\lambda_{1}\right|<1$ and $\left|\lambda_{2}\right|>1$ or $\left|\lambda_{1}\right|>1$ and $\left|\lambda_{2}\right|<1$,

iv) non-hyperbolic if either $\left|\lambda_{1}\right|=1$ or $\left|\lambda_{2}\right|=1$.

To investigate the stability of the one nontrivial fixed point of the system (1.2), we give the following lemma.

Lemma $2.2([10,17])$. Assume $F(\lambda)=\lambda^{2}+B \lambda+C$, where $B$ and $C$ are two real constants and let $F(1)>0$. Suppose that $\lambda_{1}$ and $\lambda_{2}$ are two roots of $F(\lambda)=0$. Then the following statements hold

i) $\left|\lambda_{1}\right|<1$ and $\left|\lambda_{2}\right|<1$ if and only if $F(-1)>0$ and $C<1$;

ii) $\left|\lambda_{1}\right|>1$ and $\left|\lambda_{2}\right|>1$ if and only if $F(-1)>0$ and $C>1$;

iii) $\left|\lambda_{1}\right|<1$ and $\left|\lambda_{2}\right|>1$, or $\left|\lambda_{1}\right|>1$ and $\left|\lambda_{2}\right|<1$, if and only if $F(-1)<0$,

iv) $\lambda_{1}$ and $\lambda_{2}$ are a pair of conjugate complex roots and $\left|\lambda_{1}\right|=\left|\lambda_{2}\right|=1$ if and only if $B^{2}-4 C<0$ and $C=1$.

The stability of the coexistence fixed point $E^{*}=\left(\frac{\theta r_{1}^{2}}{r_{2} \varepsilon\left(\alpha \varepsilon+r_{1}\right)}, \frac{r_{1}}{\varepsilon}\right)$ can be determined by analyzing the following matrix:

$$
J\left(E^{*}\right)=\left(\begin{array}{cc}
1 & -\frac{\delta \theta r_{1}^{2}}{r_{2}\left(\alpha \varepsilon+r_{1}\right)} \\
\frac{\delta r_{2}^{2}\left(\alpha \varepsilon+r_{1}\right)}{r_{1} \theta} & 1-\delta r_{2}\left(\frac{2 \alpha \varepsilon+r_{1}}{\alpha \varepsilon+r_{1}}\right)
\end{array}\right) .
$$

The characteristic polynomial associated with the map (1.2) at $E^{*}$ is written by

$$
F(\lambda)=\lambda^{2}-\operatorname{tr} J\left(E^{*}\right) \lambda+\operatorname{det} J\left(E^{*}\right),
$$

where

$$
\operatorname{tr} J\left(E^{*}\right)=2-\delta r_{2}\left(\frac{2 \alpha \varepsilon+r_{1}}{\alpha \varepsilon+r_{1}}\right), \quad \operatorname{det} J\left(E^{*}\right)=1+\delta^{2} r_{1} r_{2}-\delta r_{2}\left(\frac{2 \alpha \varepsilon+r_{1}}{\alpha \varepsilon+r_{1}}\right) .
$$

Since $\delta>0, r_{1}>0$ and $r_{2}>0$, then

On the other hand

$$
F(1)=\delta^{2} r_{1} r_{2}>0
$$

$$
F(-1)=\delta^{2} r_{1} r_{2}-2 \delta r_{2}\left(\frac{2 \alpha \varepsilon+r_{1}}{\alpha \varepsilon+r_{1}}\right)+4
$$

Let's write $\phi(\delta)=\delta^{2} r_{1} r_{2}-2 \delta r_{2}\left(\frac{2 \alpha \varepsilon+r_{1}}{\alpha \varepsilon+r_{1}}\right)+4 . \Delta_{F}=4 r_{2}\left[r_{2}\left(\frac{2 \alpha \varepsilon+r_{1}}{\alpha \varepsilon+r_{1}}\right)^{2}-4 r_{1}\right]$ is the discriminant of the function $\phi(\delta)$. We assume that $\Delta_{F} \geqslant 0$. Then, the function $\phi(\delta)$ has two real roots as:

$$
\delta_{1,2}=\frac{r_{2}\left(2 \alpha \varepsilon+r_{1}\right) \pm \sqrt{\Delta}}{r_{1} r_{2}\left(\alpha \varepsilon+r_{1}\right)} .
$$

Thus if $\Delta_{F} \geqslant 0$, we have $F(-1)>0$ for $\delta<\delta_{1}$ or $\delta>\delta_{2}$ and $F(-1)<0$ for $\delta_{1}<$ $\delta<\delta_{2}$. Similarly, if $\Delta_{F}<0$, since the the function $\phi(\delta)$ has not real roots for $\forall \delta>0$, $F(-1)>0$. Moreover, it is easily seen that $\operatorname{det} J\left(E^{*}\right)>1$ for $\delta<0$ or $\delta>\frac{2 \alpha \varepsilon+r_{1}}{r_{1}\left(\alpha \varepsilon+r_{1}\right)}$ and $\operatorname{det} J\left(E^{*}\right)<1$ for $0<\delta<\frac{2 \alpha \varepsilon+r_{1}}{r_{1}\left(\alpha \varepsilon+r_{1}\right)}$.

Using Lemma 2.2, we can give the topological classification of the $E^{*}$ fixed point as follows: 
Proposition 2.3. The positive fixed point $E^{*}$ of the system (1.2) is

i) sink fixed point if one of the following conditions holds:

a) $\Delta_{F} \geqslant 0$ and $0<\delta<\frac{r_{2}\left(2 \alpha \varepsilon+r_{1}\right)-\sqrt{\Delta}}{r_{1} r_{2}\left(\alpha \varepsilon+r_{1}\right)}$,

b) $\Delta_{F}<0$ and $0<\delta<\frac{2 \alpha \varepsilon+r_{1}}{r_{1}\left(\alpha \varepsilon+r_{1}\right)}$,

ii) source fixed point if one of the following conditions holds:

a) $\Delta_{F} \geqslant 0$ and $\frac{2 \alpha \varepsilon+r_{1}}{r_{1}\left(\alpha \varepsilon+r_{1}\right)}<\delta<\frac{r_{2}\left(2 \alpha \varepsilon+r_{1}\right)+\sqrt{\Delta}}{r_{1} r_{2}\left(\alpha \varepsilon+r_{1}\right)}$,

b) $\Delta_{F}<0$ and $\delta>\frac{2 \alpha \varepsilon+r_{1}}{r_{1}\left(\alpha \varepsilon+r_{1}\right)}$

iii) saddle fixed point if the following conditions holds:

$$
\Delta_{F} \geqslant 0 \text { and } \frac{r_{2}\left(2 \alpha \varepsilon+r_{1}\right)-\sqrt{\Delta}}{r_{1} r_{2}\left(\alpha \varepsilon+r_{1}\right)}<\delta<\frac{r_{2}\left(2 \alpha \varepsilon+r_{1}\right)+\sqrt{\Delta}}{r_{1} r_{2}\left(\alpha \varepsilon+r_{1}\right)},
$$

iv) non-hyperbolic fixed point if one of the following conditions holds:

a) $\Delta_{F} \geqslant 0$ and $\delta=\frac{r_{2}\left(2 \alpha \varepsilon+r_{1}\right) \pm \sqrt{\Delta}}{r_{1} r_{2}\left(\alpha \varepsilon+r_{1}\right)}$ and $\delta \neq \frac{\left(2 \alpha \varepsilon+r_{1}\right)}{r_{2}\left(\alpha \varepsilon+r_{1}\right)}, \frac{2\left(2 \alpha \varepsilon+r_{1}\right)}{r_{2}\left(\alpha \varepsilon+r_{1}\right)}$,

b) $\Delta_{F}<0$ and $\delta=\frac{2 \alpha \varepsilon+r_{1}}{r_{1}\left(\alpha \varepsilon+r_{1}\right)}$,

where $\Delta=r_{2}\left[r_{2}\left(2 \alpha \varepsilon+r_{1}\right)^{2}-4 r_{1}\left(\alpha \varepsilon+r_{1}\right)^{2}\right]$.

\section{Neimark-Sacker bifurcation analysis}

In this section, choosing the step size $\delta$ as a bifurcation parameter, a detailed bifurcation analysis is being performed at the fixed point $E^{*}$ of the system (1.2). Also, the direction of Neimark-Sacker bifurcation is evaluated.

It is clear that the eigenvalues of the Jacobian matrix $J\left(E^{*}\right)$ are a pair of conjugate complex numbers with module one, when the condition from Proposition 2.3 iv.b). Let's

$$
N S B_{E^{*}}=\left\{r_{1}, r_{2}, \delta, \theta, \varepsilon, \alpha \in(0,+\infty): \delta=\frac{2 \alpha \varepsilon+r_{1}}{r_{1}\left(\alpha \varepsilon+r_{1}\right)}, \Delta_{F}<0\right\} .
$$

If the parameters lie in $N S B_{E^{*}}$, two eigenvalues of $J\left(E^{*}\right)$ are complex having modules one and Neimark-Sacker bifurcation can emerge from the fixed point $E^{*}$. The eigenvalues under these conditions are given by

$$
\lambda, \bar{\lambda}=\frac{2 b-\delta r_{2}(b+\alpha \varepsilon) \pm i \sqrt{\delta^{2} r_{2}\left(4 r_{1} b^{2}-r_{2}(\alpha \varepsilon+b)^{2}\right)}}{2 b} .
$$

If we take

$$
\delta=\frac{2 \alpha \varepsilon+r_{1}}{r_{1}\left(\alpha \varepsilon+r_{1}\right)}
$$

the eigenvalues can be rewritten as

$$
\lambda, \bar{\lambda}=\frac{2 r_{1} b^{2}-r_{2}(b+\alpha \varepsilon)^{2} \pm i \sqrt{r_{2}(b+\alpha \varepsilon)^{2}\left(4 r_{1} b^{2}-r_{2}(\alpha \varepsilon+b)^{2}\right)}}{2 r_{1} b^{2}},
$$

where $b=\alpha \varepsilon+r_{1}$.

It is clear that

$$
|\lambda|=|\bar{\lambda}|=1
$$


Because of $\delta \in N S B_{E^{*}}$, we obtain

$$
\left.\frac{d|\lambda(\delta)|}{d \delta}\right|_{\delta=\frac{2 \alpha \varepsilon+r_{1}}{r_{1}\left(\alpha \varepsilon+r_{1}\right)}}=r_{2}\left(\frac{b+\alpha \varepsilon}{b}\right) \neq 0 .
$$

Also, if

$$
\operatorname{tr} J\left(E^{*}\right) \neq 0,-1 \text { namely } \delta r_{2}\left(\frac{b+\alpha \varepsilon}{b}\right) \neq 2,3
$$

then

$$
\lambda^{k}(\delta) \neq 1 \text { for } k=1,2,3,4 .
$$

is satisfied.

Let $q, p \in \mathbb{C}^{2}$ are two eigenvectors of $J\left(\delta_{N S B_{E^{*}}}\right)$ and transposed matrix $J^{T}\left(\delta_{N S B_{E^{*}}}\right)$ corresponding to $\lambda$ and $\bar{\lambda}$, respectively. By simple calculation we get

$$
q \sim\left(1, \frac{r_{2}\left(r_{2}(b+\alpha \varepsilon)-i \sqrt{K}\right)}{2 \theta r_{1}^{2}}\right)^{T}
$$

and

$$
p \sim\left(1,-\frac{r_{1} \theta\left(r_{2}(b+\alpha \varepsilon)+i \sqrt{K}\right)}{2 r_{2}^{2} b^{2}}\right)^{T},
$$

where $K=r_{2}\left(4 r_{1} b^{2}-r_{2}(b+\alpha \varepsilon)^{2}\right)$.

When the normalization $\langle p, q>=1$ is made, the normalized vectors can be obtained as follows

$$
q=\left(\begin{array}{c}
1 \\
\frac{r_{2}\left(r_{2}(b+\alpha \varepsilon)-i \sqrt{K}\right)}{2 \theta r_{1}^{2}}
\end{array}\right)
$$

and

$$
p=\left(\begin{array}{c}
\frac{4 r_{1}^{2} b^{4}\left[2 r_{1} b^{2}-r_{2}(b+\alpha \varepsilon)^{2}+i(b+\alpha \varepsilon) \sqrt{K}\right]}{16 r_{1}^{2} b^{4}-(b+\alpha \varepsilon)^{2}\left(K-8 r_{1}^{2} b^{2}+r_{1} r_{2}^{2}(b+\alpha \varepsilon)\right)} \\
-\frac{2 r_{1} \theta b^{2}}{r_{2}^{2}}\left[\frac{(b+\alpha \varepsilon) 2 r_{1} r_{2} b^{2}-r_{1}^{2}(b+\alpha \varepsilon)^{2}-K}{L}\right]
\end{array}\right)
$$

where $L=16 r_{1}^{2} b^{4}-(b+\alpha \varepsilon)^{2}\left[K-8 r_{1}^{2} b^{2}+r_{1} r_{2}^{2}(b+\alpha \varepsilon)\right]$. Now, let us consider

$$
x_{t}=N_{t}-\frac{\theta r_{1}^{2}}{r_{2} \varepsilon\left(\alpha \varepsilon+r_{1}\right)}, y_{t}=P_{t}-\frac{r_{1}}{\varepsilon} .
$$

So the fixed point $E^{*}$ is shifted to the point $(0,0)$. From Taylor expansion, the system (1.2) becomes following form:

$$
\left\{\begin{array}{l}
x_{t+1}=x_{t}-\frac{\delta \theta r_{1}^{2}}{r_{2}\left(\alpha \epsilon+r_{1}\right)} y_{t}+F_{1}\left(x_{t}, y_{t}\right) \\
y_{t+1}=\left(\alpha \epsilon+r_{1}\right) \frac{\delta r_{2}^{2}}{r_{1} \theta} x_{t}+\left(1-\delta r_{2} \frac{2 \alpha \epsilon+r_{1}}{\left(\alpha \epsilon+r_{1}\right)}\right) y_{t}+F_{2}\left(x_{t}, y_{t}\right)
\end{array}\right.
$$


or

$$
\left(\begin{array}{c}
x_{t} \\
y_{t}
\end{array}\right) \rightarrow J\left(E^{*}\right)\left(\begin{array}{c}
x_{t} \\
y_{t}
\end{array}\right)+\left(\begin{array}{c}
F_{1}\left(x_{t}, y_{t}\right) \\
F_{2}\left(x_{t}, y_{t}\right)
\end{array}\right)
$$

where

$$
\begin{aligned}
F_{1}\left(x_{t}, y_{t}\right)= & -\varepsilon \delta x_{t} y_{t}+O\left(\left\|X_{t}\right\|^{4}\right) \\
F_{2}\left(x_{t}, y_{t}\right)= & -\frac{\delta \varepsilon r_{2}^{2} b^{2}}{r_{1}^{3} \theta^{2}} x_{t}^{2}-\frac{\delta \varepsilon^{2} r_{2}^{3}}{r_{1}^{4} \theta^{2}} b(\alpha \varepsilon+2 b) x_{t}^{2} y_{t} \\
& -\frac{\delta \varepsilon r_{2}}{r_{1} b^{2}}\left[b^{2}+\alpha \varepsilon(\alpha \varepsilon+b)\right] y_{t}^{2}+\frac{\delta \varepsilon r_{2}^{2}}{r_{1}^{2} \theta}(\alpha \varepsilon+2 b) x_{t} y_{t} \\
& +\frac{\delta \varepsilon^{2} r_{2}^{2}}{r_{1}^{3} \theta b}\left(b^{2}+\alpha \varepsilon(\alpha \varepsilon+b)\right) x_{t} y_{t}^{2}-\frac{\delta \varepsilon^{5} r_{2} \alpha^{3}}{r_{1}^{2} b^{3}} y_{t}^{3}+O\left(\left\|X_{t}\right\|^{4}\right),
\end{aligned}
$$

and $X_{t}=\left(x_{t}, y_{t}\right)^{T}$.

Furthermore the system (1.2) can be expressed as

$$
\left(\begin{array}{l}
x_{t+1} \\
y_{t+1}
\end{array}\right)=J\left(E^{*}\right)\left(\begin{array}{l}
x_{t} \\
y_{t}
\end{array}\right)+\frac{1}{2} B\left(x_{t}, x_{t}\right)+\frac{1}{6} C\left(x_{t}, x_{t}, x_{t}\right)+O\left(\left\|X_{t}\right\|^{4}\right),
$$

where the multilinear vector functions of $x, y, u \in \mathbb{R}^{2}$ :

$$
B(x, y)=\left(\begin{array}{c}
B_{1}(x, y) \\
B_{2}(x, y)
\end{array}\right)
$$

and

are defined by

$$
C(x, y, u)=\left(\begin{array}{l}
C_{1}(x, y, u) \\
C_{2}(x, y, u)
\end{array}\right)
$$

$$
\begin{aligned}
B_{1}(x, y)= & \left.\sum_{j, k=1}^{2} \frac{\partial^{2} F_{1}}{\partial \xi_{j} \partial \xi_{k}}\right|_{\xi=0} x_{j} y_{k}=-\delta \varepsilon\left(x_{1} y_{2}+x_{2} y_{1}\right), \\
B_{2}(x, y)= & \left.\sum_{j, k=1}^{2} \frac{\partial^{2} F_{2}}{\partial \xi_{j} \partial \xi_{k}}\right|_{\xi=0} x_{j} y_{k} \\
= & -\frac{2 \delta r_{2}^{2} \varepsilon b^{2}}{r_{1}^{3} \theta^{2}} x_{1} y_{1}-\frac{2 \delta r_{2} \varepsilon}{r_{1} b^{2}}\left(b^{2}+\alpha \varepsilon(b+\alpha \varepsilon)\right) x_{2} y_{2} \\
& +\frac{\delta \varepsilon r_{2}^{2}}{r_{1}^{2} \theta}(2 b+\alpha \varepsilon)\left(x_{1} y_{2}+x_{2} y_{1}\right), \\
C_{1}(x, y, u)= & \left.\sum_{j, k, l=1}^{2} \frac{\partial^{3} F_{1}}{\partial \xi_{j} \partial \xi_{k} \partial \xi_{l}}\right|_{\xi=0} x_{j} y_{k} u_{l}=0, \\
C_{2}(x, y, u)= & \left.\sum_{j, k, l=1}^{2} \frac{\partial^{3} F_{2}}{\partial \xi_{j} \partial \xi_{k} \partial \xi_{l}}\right|_{\xi=0} x_{j} y_{k} u_{l} \\
= & -\frac{2 \delta r_{2}^{3} \varepsilon^{2}}{r_{1}^{4} \theta^{2}} b(2 b+\alpha \epsilon)\left(x_{1} y_{1} u_{2}+x_{1} y_{2} u_{1}+x_{2} y_{1} u_{1}\right) \\
& +\frac{2 \delta r_{2}^{2} \varepsilon^{2}}{\theta r_{1}^{3} b}\left(b^{2}+\alpha \epsilon(b+\alpha \epsilon)\right)\left(x_{1} y_{2} u_{2}+x_{2} y_{1} u_{2}+x_{2} y_{2} u_{1}\right) \\
& -\frac{\delta r_{2} \varepsilon^{5} \alpha^{3}}{r_{1}^{2} b^{3}} x_{2} y_{2} u_{2} .
\end{aligned}
$$


To analyze the corresponding bifurcation, introduce the complex variable: $z$ and $\bar{z}$. $\forall X \in \mathbb{R}^{2}$ can be uniqely represented as

$$
X=z q+\overline{z q}
$$

for some $z \in \mathbb{C}$. It is clear that $z=<p, X>$.

The system (1.2) can be transformed for all sufficiently small $|\delta|$ into the form

$$
z \rightarrow \lambda_{1}(\delta) z+g(z, \bar{z}, \delta),
$$

where $\lambda(\delta)=(1+\varphi(\delta)) e^{i \theta(\delta)}$ with $\varphi\left(\delta_{N S}\right)=0$ and $g(z, \bar{z}, \delta)$ is a complex-valued smooth function of $z$ and $\bar{z}$. After Taylor expression of $g$ with respect to $(z, \bar{z})$, we obtain

$$
g(z, \bar{z}, \delta)=\sum_{k+l \geq 2} \frac{1}{k ! l !} g_{k l}(\delta) z^{k} \bar{z}^{l}
$$

where

$$
\begin{aligned}
& g_{20}\left(\delta_{N S}\right)=<p, B(q, q)> \\
& g_{11}\left(\delta_{N S}\right)=<p, B(q, \bar{q})> \\
& g_{02}\left(\delta_{N S}\right)=<p, B(\bar{q}, \bar{q})> \\
& g_{21}\left(\delta_{N S}\right)=<p, C(q, q, \bar{q})>.
\end{aligned}
$$

The direction of the Neimark-Sacker bifurcation is computed by $\operatorname{sign}\left(a\left(\delta_{N S}\right)\right)$. The coefficient $a\left(\delta_{N S}\right)$ is given via

$$
\begin{aligned}
a\left(\delta_{N S}\right)= & \operatorname{Re}\left(\frac{e^{-i \theta\left(\delta_{N S}\right)}}{2} g_{21}\right) \\
& -\operatorname{Re}\left(\frac{\left(1-2 e^{i \theta\left(\delta_{N S}\right)}\right) e^{-2 i \theta\left(\delta_{N S}\right)}}{2\left(1-e^{i \theta\left(\delta_{N S}\right)}\right)} g_{20} g_{11}\right)-\frac{1}{2}\left|g_{11}\right|^{2}-\frac{1}{4}\left|g_{02}\right|^{2}
\end{aligned}
$$

where $e^{i \theta\left(\delta_{N S}\right)}=\lambda\left(\delta_{N S}\right)$.

We get the following theorem on Neimark-Sacker bifurcation:

Theorem 3.1. If (3.6) holds, $a\left(\delta_{N S}\right) \neq 0$ and the parameter $\delta$ changes its value in small vicinity of $N S B_{E^{*}}$, then the model (1.2) passes through a Neimark-Sacker bifurcation at only fixed point $E^{*}$. Moreover if a $\left(\delta_{N S}\right)<0\left(\right.$ resp., a $\left.\left(\delta_{N S}\right)>0\right)$, then there exists a unique attracting (resp., repelling) invariant closed curve which bifurcates from $E^{*}$.

\section{Chaos control}

Chaotic behavior is very interesting non-linear phenomenon observed in many fields such as physics, biology, chemistry and engineering. The chaos is harmful to systems as it can lead them to be unstable and make undesirable behaviors. So chaos control methods are required to decrease or eliminate the harmful chaotic effect. Chaotic dynamics must be controlled according to a regular orbit, such as a periodic orbit or a fixed point to improve the performance of the system under consideration. Chaos control refers to deliberately manipulating the chaotic dynamic behavior of some complex nonlinear systems. There are numerous methods in literature for chaos control such as OGY method [23,27], time-delay feed-back control method [22] and fuzzy control [9], etc. The OGY method was proposed by Ott, Greborgi and Yorke [27]. The common idea of Ott, Grebogi and Yorke consists of turning the presence of chaos into an advantage. In fact, the system can be stabilized on a certain unstable periodic orbit embedded in a strange attractor by applying a small time-dependent feedback perturbation to some accessible parameter or variable system. 
The periodic orbit is maintained, but its stability is changed so that the orbit stays close to the unstable periodic orbit. This control strategy is known as the OGY method. Also see $[23,25,27]$ for details related to OGY method. In this section, we apply the OGY control method to stabilize chaotic orbits an unstable fixed point of the system (1.2).

Constructing the controlled system is as follows:

$$
\begin{aligned}
& N_{t+1}=N_{t}+\delta N_{t}\left(r_{1}-\varepsilon P_{t}\right)=f\left(N_{t}, P_{t}, r_{1}\right) \\
& P_{t+1}=P_{t}+\delta P_{t}\left(r_{2}-\theta \frac{P_{t}}{N_{t}} \frac{P_{t}}{\left(\alpha+P_{t}\right)}\right)=g\left(N_{t}, P_{t}, r_{1}\right)
\end{aligned}
$$

where $r_{1}$ is taken as controlling parameter. Moreover, $r_{1}$ is restricted to line in some small interval $\left|r_{1}-r_{1_{0}}\right|<\mu$ with $\mu>0$ and $r_{1_{0}}$ denotes the nominal value belong to chaotic region. Assume that $\left(N^{*}, P^{*}\right)$ be unstable fixed point of the system (1.2) in chaotic region produced by the emergence of Neimark-Sacker bifurcation, then the system (4.1) can be approximated in the neigborhood of the unstable fixed point $\left(N^{*}, P^{*}\right)$ by the following linear map:

$$
\left[\begin{array}{c}
N_{t+1}-N^{*} \\
P_{t+1}-P^{*}
\end{array}\right] \approx A\left[\begin{array}{c}
N_{t}-N^{*} \\
P_{t}-P^{*}
\end{array}\right]+B\left[r_{1}-r_{1_{0}}\right]
$$

where

$$
A=\left[\begin{array}{ll}
\frac{\partial f\left(N^{*}, P^{*}, r_{1}\right)}{\partial N_{t}} & \frac{\partial f\left(N^{*}, P^{*}, r_{1_{0}}\right)}{\partial P_{t}} \\
\frac{\partial g\left(N^{*}, P^{*}, r_{1_{0}}\right)}{\partial N_{t}} & \frac{\partial g\left(N^{*}, P^{*}, r_{1_{0}}\right)}{\partial P_{t}}
\end{array}\right],
$$

and

$$
B=\left[\begin{array}{c}
\frac{\partial f\left(N^{*}, P^{*}, r_{1_{0}}\right)}{\partial r_{1}} \\
\frac{\partial f\left(N^{*}, P^{*}, r_{1_{0}}\right)}{\partial r_{1}}
\end{array}\right]=\left[\begin{array}{c}
\delta \theta r_{1_{0}}^{2} \\
r_{2} \varepsilon b \\
0
\end{array}\right] .
$$

On the other hand the system (4.1) is controllable provided that the following matrix

$$
\begin{aligned}
C & =[B: A B] \\
& =\left[\begin{array}{cc}
\frac{\delta \theta r_{1_{0}}^{2}}{r_{2} \varepsilon b} & \frac{\delta \theta r_{1_{0}}^{2}}{r_{2} \varepsilon b} \\
0 & \frac{\delta^{2} r_{2} r_{1_{0}}}{\varepsilon}
\end{array}\right] .
\end{aligned}
$$

Since all parameters are positive, the rank of $C$ is 2 . Furthermore, $\frac{\delta^{2} r_{2} r_{0}}{\varepsilon} \neq 0$ implies that the system (4.1) is always controllable. Now, we suppose that $\left[r_{1}-r_{1_{0}}\right]=$ $-K\left[\begin{array}{c}N_{t}-N^{*} \\ P_{t}-P^{*}\end{array}\right]$, where $K=\left[\begin{array}{ll}\rho_{1} & \rho_{2}\end{array}\right]$, then the system (4.1) can be written as follows:

$$
\left[\begin{array}{c}
N_{t+1}-N^{*} \\
P_{t+1}-P^{*}
\end{array}\right] \approx[A-B K]\left[\begin{array}{c}
N_{t}-N^{*} \\
P_{t}-P^{*}
\end{array}\right]
$$

The corresponding controller can be rewritten as:

$$
\begin{aligned}
& N_{t+1}=N_{t}+\delta N_{t}\left(\left(r_{1_{0}}-\rho_{1}\left(N_{t}-N^{*}\right)-\rho_{2}\left(P_{t}-P^{*}\right)\right)-\varepsilon P_{t}\right), \\
& P_{t+1}=P_{t}+\delta P_{t}\left(r_{2}-\theta \frac{P_{t}}{N_{t}} \frac{P_{t}}{\left(\alpha+P_{t}\right)}\right) .
\end{aligned}
$$

Furthermore, the fixed point $\left(N^{*}, P^{*}\right)$ of $(4.1)$ is locally asymptotically stable if and only if both eigenvalues of the matrix $A-B K$ lie in an open unit disk. The Jacobian matrix $A-B K$ of the controlled system (4.1) can be written as follow:

$$
A-B K=\left[\begin{array}{cc}
1-\frac{\delta^{2} \theta \rho_{1} r_{0}}{r_{2} \varepsilon b} & -\frac{\delta \theta r_{1}^{2}}{b r_{2}}\left(1+\frac{\rho_{2}}{\varepsilon}\right) \\
\frac{\delta b r_{2}^{2}}{\theta r_{1}} & 1-\frac{\delta r_{2}(b+\alpha \varepsilon)}{b}
\end{array}\right] .
$$

The characteristic equation of the Jacobian matrix $A-B K$ is given by 


$$
\begin{aligned}
P(\lambda)= & \lambda^{2}-\left(2-\frac{\delta \theta \rho_{1} r_{1_{0}}^{2}}{\varepsilon b r_{2}}-\frac{\delta r_{2}(b+\alpha \varepsilon)}{b}\right) \lambda \\
& +\frac{1}{r_{2} \varepsilon b^{2}}\left(\delta^{2} r_{1_{0}} r_{2}\left(r_{2} b^{2}\left(\rho_{2}+\varepsilon\right)+\theta r_{1_{0}} \rho_{1}(b+\alpha \varepsilon)\right)\right) .
\end{aligned}
$$

Let $\lambda_{1}$ and $\lambda_{2}$ are the eigenvalues of characteristic equation (4.1), then

$$
\begin{aligned}
& \lambda_{1}+\lambda_{2}=2+r_{2}(\delta-2 \theta)-\frac{\delta^{2} \rho_{1} r_{1_{0}}}{r_{2} \varepsilon} \\
& \lambda_{1} \lambda_{2}=1+r_{2}(\delta-2 \theta)-\frac{\delta^{2} r_{2} r_{1_{0}}}{\varepsilon}\left(\frac{\rho_{1}}{r_{2}^{2}}\left(1+r_{2}(\delta-2 \theta)\right)+\theta\left(1+\frac{\rho_{2}}{\varepsilon}\right)\right)
\end{aligned}
$$

are valid.

In order to obtain the lines of marginal stability we must solve equations $\lambda_{1}= \pm 1$ and $\lambda_{1} \lambda_{2}=1$. These restrictions make sure that $\lambda_{1}$ and $\lambda_{2}$ have absolute less than 1.Assume that $\lambda_{1} \lambda_{2}=1$, Eq. (4.7) implies that:

$$
L_{1}:=-\frac{\delta \theta \rho_{1} r_{1_{0}}^{2}}{r_{2} \varepsilon b^{2}}\left(\delta r_{2}(b+\alpha \varepsilon)+b\right)+\frac{\delta^{2} r_{2}^{2} \rho_{2} r_{1_{0}}}{\varepsilon}+\delta r_{2}\left(\delta r_{1_{0}}-1-\frac{\alpha \varepsilon}{b}\right)=0
$$

Moreover, we suppose that $\lambda_{1}=1$, then (4.6)-(4.7) yield that:

$$
L_{2}:=\frac{\delta^{2} \theta \rho_{1} r_{1_{0}}^{2}}{\varepsilon b^{2}}(b+\alpha \varepsilon)+\frac{\delta \theta r_{2} \rho_{2} r_{1_{0}}}{\varepsilon}+\delta^{2} r_{2} r_{1_{0}}=0
$$

Finally, taking $\lambda_{1}=-1$ and using equation (4.6)-(4.7) we get

$$
L_{3}:=-\frac{\delta \theta \rho_{1} r_{1_{0}}^{2}}{r_{2} \varepsilon b^{2}}\left(\delta\left(r_{2} b+\alpha \varepsilon\right)-2 b\right)+\frac{\delta^{2} r_{2} \rho_{2} r_{1_{0}}}{\varepsilon}+\delta^{2} r_{2} r_{1_{0}}-\frac{2 \delta r_{2}}{\varepsilon}\left(1+\frac{\alpha \varepsilon}{b}\right)+4=0
$$

Then, stable eigenvalues lie within the triangular region in $\rho_{1} \rho_{2}$ plane bounded by the straight lines $L_{1}, L_{2}, L_{3}$ for particular parametric values.

\section{Numerical simulations}

In this section, all the previous analytical evidences are verified with the help of numerical simulations performed via Matlab. We present the bifurcation diagrams, phase portraits, and maximum Lyapunov exponents for the system (1.2) with Allee effect. Thus, numerical simulations have displayed valuable and meaningful dynamical behaviors, which show that the model can eventually lead to chaos by Neimark-Sacker bifurcation.

Example 5.1. For the parameter values $r_{1}=4, r_{2}=14, \delta=0.23, \theta=50, \varepsilon=0.4$, $\alpha=0.23$ and initial condition $\left(N_{0}, P_{0}\right)=(34,9.5)$, the positive fixed point of the model $(1.2)$ is obtained as $\left(N^{*}, P^{*}\right)=(34.91132522,10)$. From Figure 1, the fixed point $\left(N^{*}, P^{*}\right)$ of the system (1.2) is local asymptotically stable where the graphs represent $P(t)$ and $N(t)$ populations, respectively. 

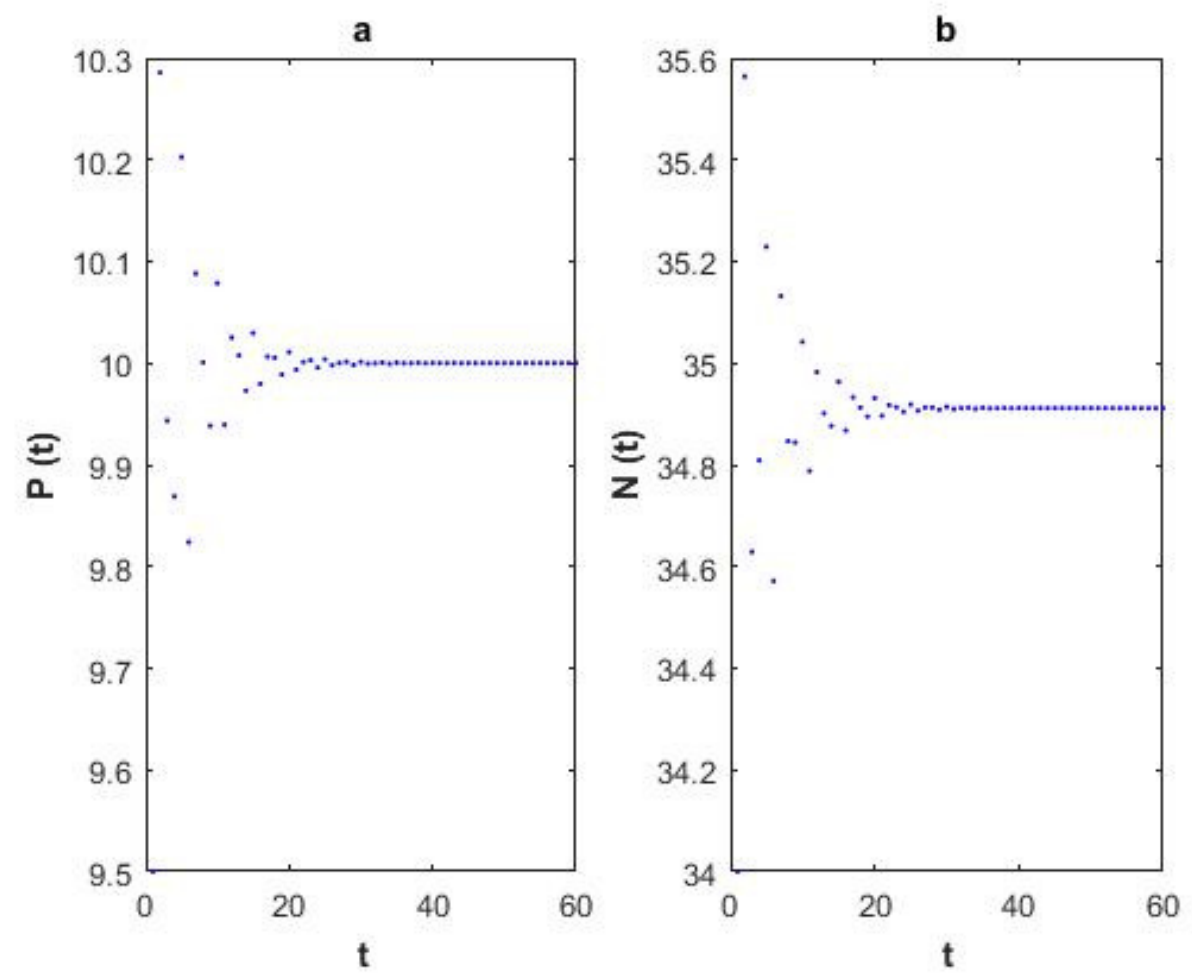

Figure 1. A stable fixed point for the system (1.2) for $r_{1}=4, r_{2}=14, \delta=$ $0.23, \theta=50, \varepsilon=0.4, \alpha=0.23$ and initial condition $\left(N_{0}, P_{0}\right)=(34,9.5)$.

Example 5.2. For the parameters values $r_{1}=4, r_{2}=14, \theta=50, \varepsilon=0.4, \delta_{N S}=$ $0.2556207234, \alpha=0.23$, the positive fixed point $\left(N^{*}, P^{*}\right)$ of the model $(1.2)$ is evaluated as $\left(N^{*}, P^{*}\right)=(34.91132522,10)$. Because of computing the coefficients of normal form, we transform to point $(0,0)$ to fixed point $\left(N^{*}, P^{*}\right)$ by change of variables,

$$
\begin{aligned}
& x=N-34.91132522 \\
& y=P-10 .
\end{aligned}
$$

So, the model (1.2) converts to

$$
\begin{gathered}
x_{t+1}=x_{t}-0.1022482894\left(x_{t}+34.91132522\right) y_{t} \\
y_{t+1}=y_{t}+0.2556207234 \frac{\left(y_{t}+10\right)\left(14\left(y_{t}+10.23\right)-50\left(y_{t}+10\right)^{2}\right)}{\left(x_{t}+34.91132522\right)\left(y_{t}+10.23\right)} .
\end{gathered}
$$

Using the above parameters, we get

$$
J\left(N^{*}, P^{*}\right)=\left[\begin{array}{cc}
1 & -3.569623284 \\
0.9579667646 & -2.419540691
\end{array}\right] .
$$

The eigenvalues are obtained as follows:

$$
\lambda_{1,2}=-0.7097703455 \pm 0.7044613785 i \text {. }
$$

Let $q, p \in C^{2}$ be complex eigenvectors corresponding to $\lambda_{1,2}$ respectively

$$
q \sim(-0.7353714178+1.784790882 i, i)^{T},
$$

and

$$
p \sim(0.1973489417+0.4789778108 i,-i)^{T} .
$$

To obtain the normalization $\langle p, q\rangle=1$, we can take normalized vectors as

$$
q=(-0.7353714178+1.784790882 i, i)^{T}
$$


and

$$
p=\left(-0.6799285207+3.62648408410^{-10} i\right)^{T} .
$$

By using the formula (3.17-3.20) the coefficients of the normal of the system (1.2) can be computed as follows:

$$
\begin{aligned}
& g_{20}(\delta)=0 \\
& g_{11}(\delta)=-0.05112414463-0.1349731779 i \\
& g_{02}(\delta)=0.2627353398+0.11956541671 i \\
& g_{21}(\delta)=0
\end{aligned}
$$

From $(3.21)$, the critical part is obtained as $a\left(\delta_{N S}\right)=-0.03124715537<0$. Therefore, a supercritical Neimark-Sacker bifurcation occurs at $\delta_{N S}=0.2556207234$. The bifurcation diagram and the phase portrait of the system (1.2) are shown in Figure 2 and Figure 3.

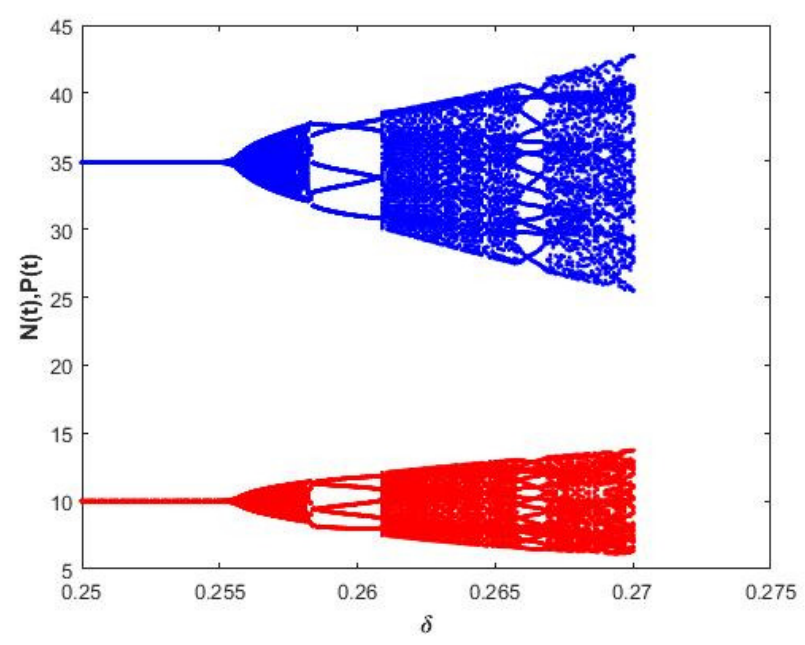

(a)

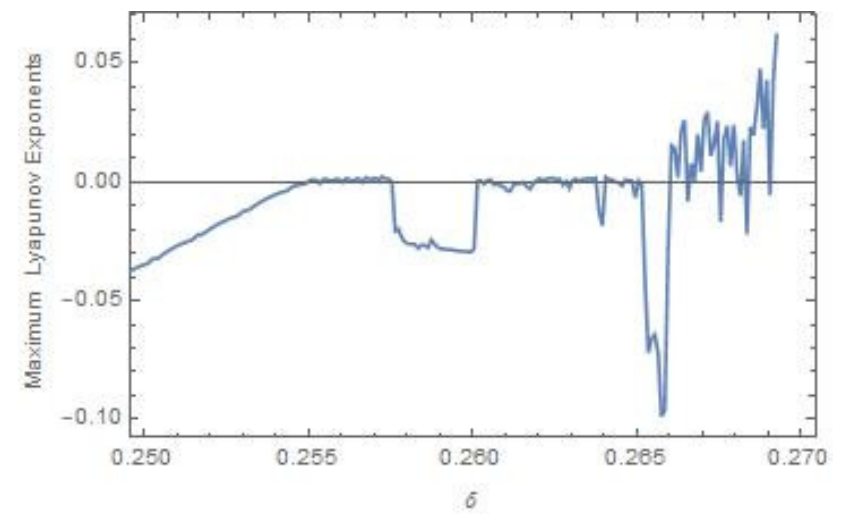

(b)

Figure 2. (a) Bifurcation diagram (b) Maximum Lyapunov exponents for the system (1.2) for values of $r_{1}=4, r_{2}=14, \delta=(0.25,0.27), \theta=50, \varepsilon=0.4, \alpha=0.23$ and initial condition $\left(N_{0}, P_{0}\right)=(34,9.5)$. 

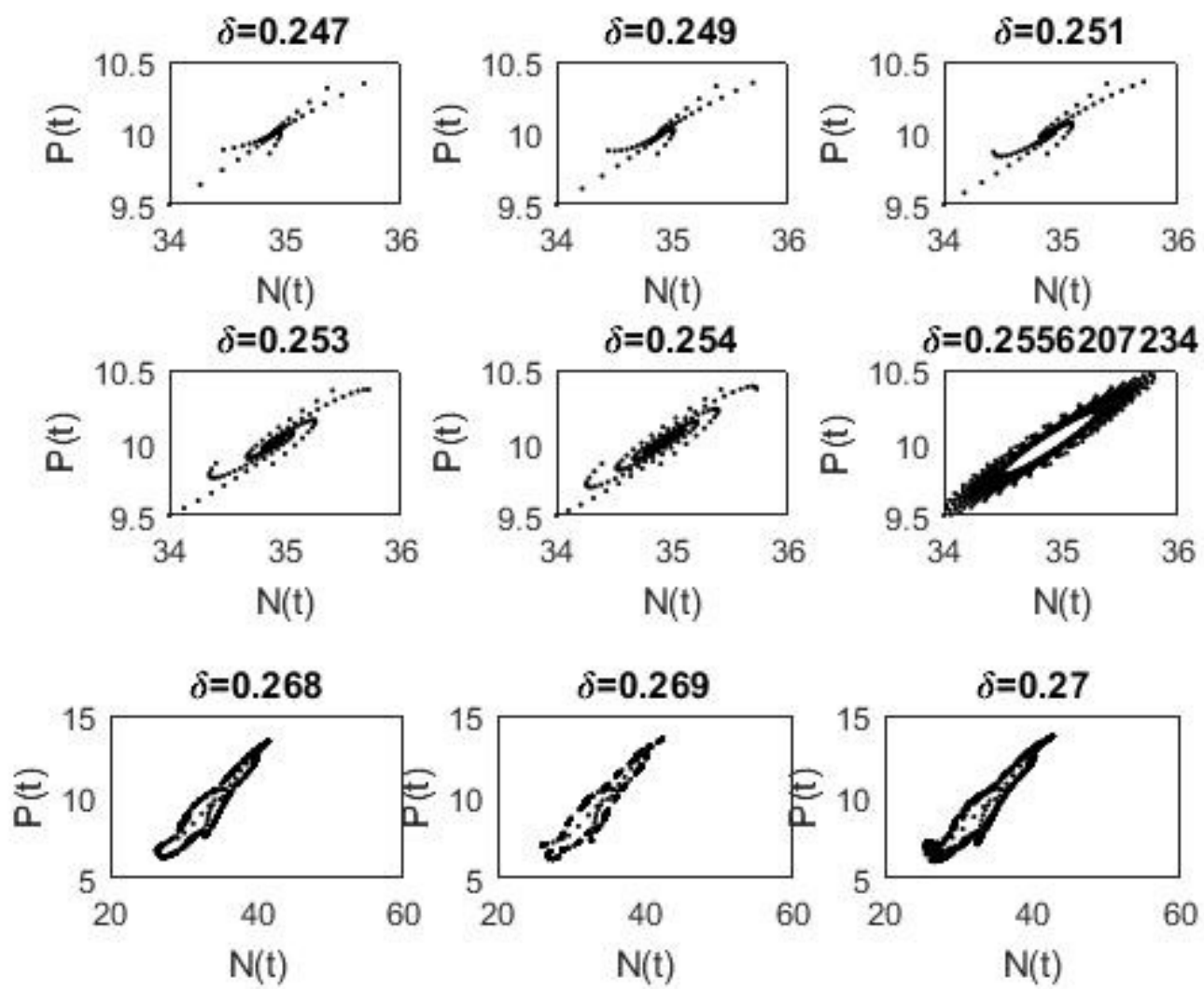

Figure 3. Phase portraits of the system (1.2) for different values of $\delta$.

Example 5.3. Let $r_{1 N S}=3.934043156, r_{2}=14, \delta=0.26, \theta=50, \varepsilon=0.4, \alpha=0.23, r_{1} \in$ $[3.8,4.15]$ and $\left(N_{0}, P_{0}\right)=(34,9.5)$, then system $(1.2)$ undergoes Neimark-Sacker bifurcation. For these parametric values, bifurcation diagram, and corresponding maximum Lyapunov exponents are plotted in Figure 4. In order to discuss the OGY feedback control method for system (1.2), we take $r_{1_{0}}=4$.147. System (1.2) has unique positive fixed point $\left(N^{*}, P^{*}\right)=(36.22318480,10.36750000)$. Then corresponding controlled system is given by:

$$
\begin{aligned}
& N_{t+1}=N_{t}+0.26 N_{t}\left(\left(4.147-\rho_{1}\left(N_{t}-36.22318480\right)-\rho_{2}\left(P_{t}-10.36750000\right)\right)-0.4 P_{t}\right), \\
& P_{t+1}=P_{t}+0.26 P_{t}\left(14-50 \frac{P_{t}}{N_{t}} \frac{P_{t}}{\left(0.23+P_{t}\right)}\right) .
\end{aligned}
$$

where $K=\left[\rho_{1} \rho_{2}\right]$ be matrix and $\left(N^{*}, P^{*}\right)=(36.22318480,10.36750000)$ is unstable fixed point of the system (1.2). We have

$$
\begin{aligned}
A & =\left[\begin{array}{cc}
1 & -3.767211220 \\
1.041810658 & -2.718999764
\end{array}\right], \\
B & =\left[\begin{array}{c}
9.418028049 \\
0
\end{array}\right]
\end{aligned}
$$

and

$$
\begin{aligned}
C & =[B: A B] \\
& =\left[\begin{array}{cc}
9.418028049 & 10.22404907 \\
0 & 8.146575000
\end{array}\right] .
\end{aligned}
$$


Then it is easy to check that rank of $C$ matrix is 2 . Therefore the system (5.2) is controllable. Then, Jacobian matrix $A-B K$ of the controlled system (5.2) is given by

$$
A-B K=\left[\begin{array}{cc}
1-9.418028049 \rho_{1} & -3.767211220-9.418028049 \rho_{2} \\
1.041810658 & -2.718999764
\end{array}\right]
$$

Moreover, the lines $L_{1}, L_{2}$ and $L_{3}$ for marginal stability are given by:

$$
\begin{aligned}
L_{1}= & 0.2057210360+25.60761604 \rho_{1}+9.811801999 \rho_{2}=0, \\
L_{2}= & 3.924720800+35.02564409 \rho_{1}+9.811801999 \rho_{2}=0, \\
& \text { and } \\
L_{3}= & 0.4867212720+16.18958799 \rho_{1}+9.811801999 \rho_{2}=0 .
\end{aligned}
$$

Then, the stable triangular region bounded by marginal lines $L_{1}, L_{2}$ and $L_{3}$ for the controlled system (5.2) is shown in Figure 5.

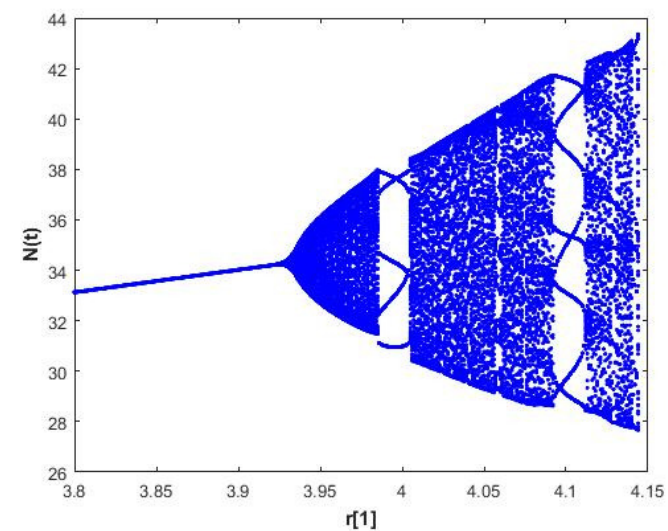

(a)

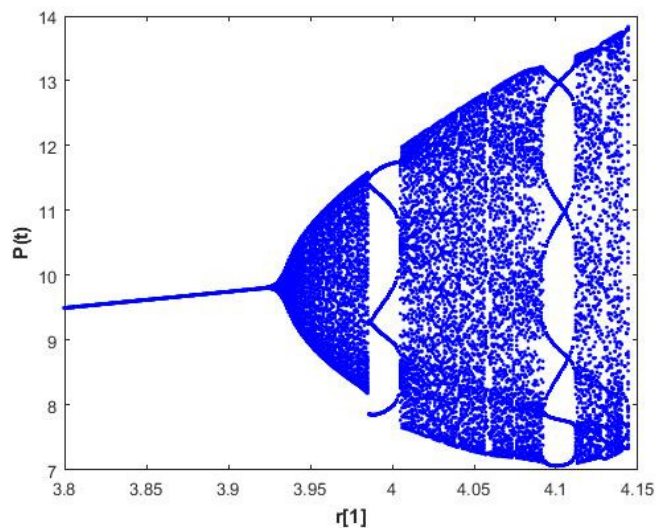

(b)

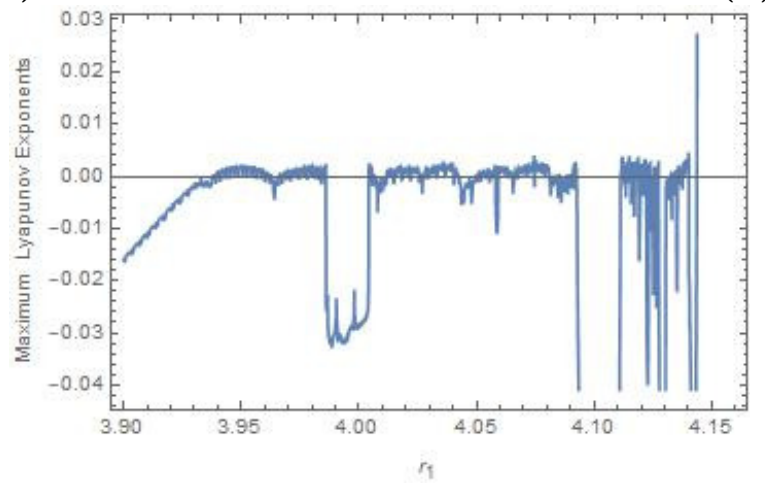

(c)

Figure 4. Bifurcation diagrams and MLE for the system (1.2) for values of $r_{2}=$ $12 \delta=0.27, \theta=50, \varepsilon=0.4, r_{1}=(3.6,4.5)$, and initial condition $\left(N_{0}, P_{0}\right)=$ (34,8.1). (a) Bifurcation diagram for $N_{t}$ (b) Bifurcation diagram for $P_{t}$ (c) Maximum Lyapunov exponents. 


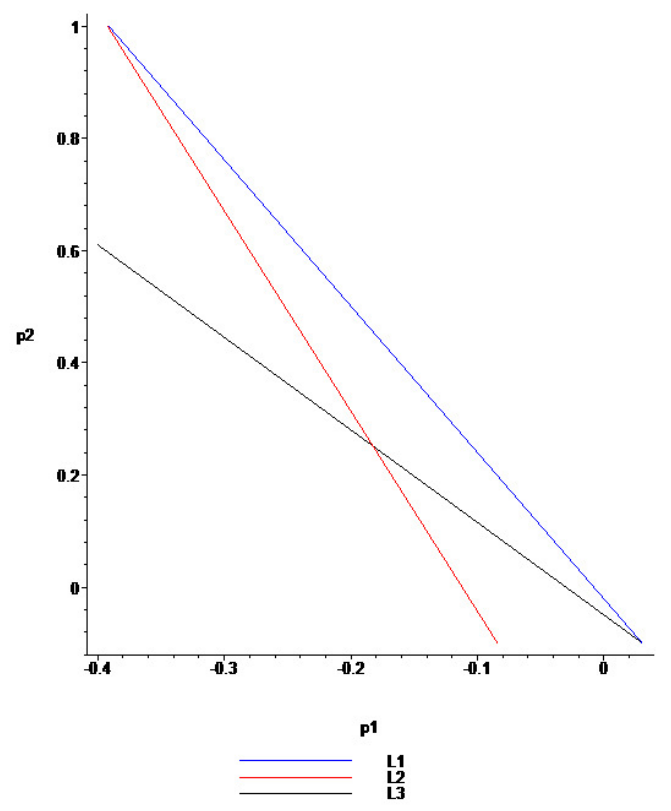

Figure 5. Triangular stability region bounded by $L_{1}, L_{2}$ and $L_{3}$ for the controlled system (5.1).

\section{Discussions}

In this paper, we have introduced a discrete-time predator-prey model with Allee effect on predator population. The existence and topological classification of the coexistence fixed point $E^{*}$ of the system (1.2) have been shown. The system (1.2) undergoes Neimark-Sacker bifurcation when the parameters $r_{1}, r_{2}, \delta, \theta, \varepsilon, \alpha$ go through the curves $N S B_{E^{*}}=\left\{r_{1}, r_{2}, \delta, \theta, \varepsilon, \alpha \in(0,+\infty): \delta=\frac{2 \alpha \varepsilon+r_{1}}{r_{1}\left(\alpha \varepsilon+r_{1}\right)}, \Delta_{F}<0\right\}[8,19,33]$. In other words, attracting invariant closed curves appears. From the viewpoint of biologically, attractive invariable closed curves bifurcated from the Neimark-Sacker bifurcation mean that predator-prey population will coexist under the periodic or quasiperiodic oscillations for a long time. Moreover, for supporting theoretical results obtained, some figures are presented by using MATLAB and MAPLE programmes. The drawn phase potraits imply invariant curve or behave chaotic. In that case, we can say that more complex dynamics are observed than in the continuous time systems.

In Figure 1 and Figure 2, stability and bifurcation diagrams of the system (1.2) are given for some parameter values, respectively. In Figure 2 , we show that $E^{*}$ is stable for $\delta<0.2556207234$ and losses its stability at $\delta=0.2556207234$ and an attracting invariant curve appears if $\delta>0.2556207234$. In Figure 3, the phase portraits of the system (1.2) for the different values of the $\delta$ are plotted. We can say that the parameter $\delta$ has a strong effect for the stability of the system (1.2) and on interaction of two populations. In Figure $4 \mathrm{a}$ and $4 \mathrm{~b}$, the bifurcation process and the emergence of a closed invariant curve are shown. In Figure 4c we calculated maximum Lyapunov exponents to illustrate the stability of the fixed point $E^{*}$. When $r_{1}$ lies in a small neighborhood of the corresponding exponents are positive, which implies the occurrence of the chaotic phenomena.

To sum up, from a biological point of view, introduction of Allee effect in the model (1.2) improves the stability results, enriches the dynamics of the model, keeps the population densities in balance and makes the model closer to reality. 
Acknowledgment. This work is supported by the Scientific Research Project Fund of Sivas Cumhuriyet University under the project number EĞT-067.

\section{References}

[1] H.N. Agiza, E.M. Elabbasy, H. El-Metwally and A.A. Elsandany, Chaotic dynamics of a discrete prey-predator model with Holling type II, Nonlinear Anal. Real World Appl. 10, 116-129, 2009.

[2] W.C. Allee, Animal Aggregations, a Study in General Sociology, University of Chicago Press, Chicago, IL, 1931.

[3] S. Biswas, D. Pal, G.S. Mahapatra and G.P. Samanta, Dynamics of a prey-predator system with herd behaviour in both and strong Allee effect in prey, Biophysics 65 (5), 826-835, 2020.

[4] L. Cheng and H. Cao, Bifurcation analysis of a discrete-time ratio-dependent predatorprey model with the Allee effect, Commun. Nonlinear Sci. Numer. Simul. 38, 288-302, 2016.

[5] Q. Din, Neimark-Sacker bifurcation and chaos control in Hassel-Varley model, J. Difference Equ. Appl. 23 (4), 741-762, 2016.

[6] Q. Din, Complexity and choas control in a discrete-time prey-predator model, Commun Nonlinear Sci. Numer. Simul. 49, 113-134, 2017.

[7] Q. Din, Bifurcation analysis and chaos control in a Host-parasitoid model, Mat. Methods Appl. Sci. 40, 5391-5406, 2017.

[8] S.N. Elaydi, An Introduction to Difference Equations, Springer-Verlag, New York, USA, 1996.

[9] C.B. Fu, A.H. Tian, K.N. Yu, Y.H. Lin and H.T. Yau, Analyses and control of chaotic behavior in DC-DC converters, Math. Probl. Eng. 2018, Article ID 7439137, 2018.

[10] Z. He and X. Lai, Bifurcation and chaotic behavior of a discrete-time predator-prey system, Nonlinear Anal. Real World Appl. 12, 403-417, 2011.

[11] Z. Hu, Z. Teng and L. Zhang, Stability and bifurcation analysis of a discrete predatorprey model with nonmonotonic functional response, Nonlinear Anal. Real World Appl. 12, 2356-2377, 2011.

[12] P.K. Jha and S. Ghorai, Stability of prey-predator model with Holling type response function and selective harvesting, J. Appl. Comput. Math. 6 (3), 2017.

[13] F. Kangalgil and S. Isık, Controlling chaos and Neimark-Sacker bifurcation discretetime predator-prey system, Hacet. J. Math. Stat. 49 (5), 1761-1776, 2020.

[14] Ş. Kartal, Dynamics of a plant-herbivore model with differential-difference equations, Cogents Math. 3, 1136198, 2016.

[15] Ş. Kartal, Flip and Neimark-Sacker bifurcation in a differential equation with piecewise constant arguments model, J. Difference Equ. Appl. 23 (4), 763-778, 2017.

[16] Ş. Kartal and F. Gurcan, Global behaviour of a predator-prey like model with piecewise constant arguments, J. Biol. Dyn. 9 (1), 159-171, 2015.

[17] A.Q. Khan, Neimark-Sacker bifurcation of a two-dimensional discrete-time predatorprey model, Springer Plus 5, Article No: 5, 2016.

[18] A. Korobeinikov, A Lyapunov function for Leslie-Gower predator-prey models, Appl. Math. Lett. 14 (6), 697-699, 2001.

[19] Y.A. Kuznetsov, Elements of Applied Bifurcation Theory, 2nd edition, SpringerVerlag, New York, USA, 1998.

[20] P.H. Leslie, Some further notes on the use of matrices in population mathematics, Biometrika 35, 213-245, 1948.

[21] P.H. Leslie, A stochastic model for studying the properties of certain biological systems by numerical methods, Biometrika 45, 16-31, 1958. 
[22] S. Li and T. Chen, Nonlinear dynamics in the switched reluctance motor drive with time-delay feedback control, IEEE Texas Power and Energy Conference (TPEC), 1-6, 2019.

[23] Y. Ling and Z. Liu, An improvement and proof of OGY method, Appl. Math. Mech. 19 (1), 1-8, 1998.

[24] X. Liu and D. Xiao, Complex dynamics behaviors of a discrete-time predator-prey system, Chaos Solitons Fractals, 32, 80-94, 2007.

[25] S. Lynch, Dynamical Systems with Applications using Mathematica, Birkhauser, Boston, 2007.

[26] P.S. Mandal, U. Kumar, K. Garain and R. Sharma, Allee effect can simplify the dynamics of a prey-predator model, J. Appl. Math. Comput. 63, 739-770, 2020.

[27] E. Ott, C. Grebogi and J.A. Yorke, Controlling chaos, Phys. Rev. Lett. 64 (11), 1196-1199, 1990.

[28] S.M. Rana and U. Kulsum, Bifurcation analysis and chaos control in a discrete-time predator-prey system of Leslie type with simplified Holling type IV functional response, Discrete Dyn. Nat. Soc. 2017, Article ID 9705985, 2017.

[29] S.M. Salman, A.M. Yousef and A.A. Elsadany, Stability, bifurcation analysis and chaos control of a discrete predator-prey system with square root functional response, Chaos Solitons Fractals, 93, 20-31, 2016.

[30] M. Sen, M. Banarjee and A. Morozou, Bifurcation analysis of a ratio-dependent preypredator model with the Allee effect, Ecol. Complex. 11, 12-27, 2012.

[31] B. Tiwari and S.N. Raw, Dynamics of Leslie-Gower model with double Allee effect on prey and mutual interference among predators, Nonlinear Dyn. 103, 1229-1257, 2021.

[32] S. Wang and H. Yu, Complexity analysis of a modified pedator-prey system with Beddington-DeAngelis functional response and Allee-like effect on predator, Discrete Dyn. Nat. Soc. 2021, Article ID 5618190, 2021.

[33] S. Wiggins, Introduction to Applied Nonlinear Dynamical System and Chaos, 2, Springer-Verlag, New York, USA, 2003.

[34] S. Zhou, Y. Liu and G. Wang, The stability of predator-prey systems subject to the Allee Effects, Theor. Popul. Biol. 67, 23-31, 2005. 